\title{
The biochemical parameters in venous blood sampling by direct venipuncture and peripheral intravenous catheter
}

\author{
Fatemeh Jaafaraghaei', Monireh Aghajany-Nasab2², Mahsa Aghaei', Nazila Javadi pashaki', Ehsan Kazemnejhad \\ Leili
}

\author{
1 Nursing and Midwifery Faculty, Guilan University of Medical Sciences, Rasht, Iran \\ 2 Department of Biochemistry, Molecule and Cell Research Center, School of Medicine, Guilan University of Medical Sciences, Rasht, Iran \\ 3 Social Determinants of Health Research Center, Nursing and Midwifery Faculty, Guilan University of Medical Sciences, Rasht, Iran \\ Corresponding author: Aghajany-Nasab Monireh, (E-mail: maghajany@gums.ac.ir ) \\ (Submitted: 14 July 2020 - Revised version received: 21 July 2020 - Accepted: 08 August 2020 - Published online: 30 October 2020)
}

\begin{abstract}
Objectives: The aim of this study was to compare the results of some biochemical values in venous blood samples obtained by direct venipuncture (DV) and peripheral venous catheter (PVC).

Methods: In this cross-sectional study, 78 hospitalized patients from different wards of Poor-Sina Medical and Educational Center of Rasht in 2017 were divided into 3 equal groups, including patients who were treated with normal saline and dextrose saline and those who did not receive this solution. Two separate blood samples were obtained from each patient, one from PVC and the other from DV. The levels of sodium, potassium, creatinine and blood urea nitrogen (BUN) were measured and compared.

Results: Difference of PVC with DV in BUN was generally $0.32 \pm 3.18 \mathrm{mg} / \mathrm{dl}$; creatinine, sodium, and potassium were $0.02 \pm 0.12 \mathrm{mg} / \mathrm{dl}$, $1.00 \pm 2.49 \mathrm{mEq} / \mathrm{L}$ and $0.15 \pm 0.48 \mathrm{mEq} / \mathrm{L}$ respectively. There were no statistically significant differences between the two methods in relation to $B U N$ and creatinine $(P=0.377$ and $P=0.149$, respectively), but significant differences in levels of sodium and potassium were observed between the two blood sampling methods $(P=0.001$ and $P=0.008$, respectively).

Conclusion: According to the results of this study and other studies, PVC can be used to measure BUN and creatinine, not so for sodium and potassium. For other parameters, further investigation is needed.

Keywords: Biochemical values, Peripheral catheterization, Blood specimen collection
\end{abstract}

\section{Introduction}

Patients admitted to the hospital are exposed to various invasive procedures during their hospitalization, so one of the biggest challenges in nursing is ensuring patient comfort and pain relief, and this has been always acknowledged in the activities and policies of the health-care system. ${ }^{1}$ One of the most common invasive procedures in hospitalized patients is direct perforation of the vein to collect blood samples for diagnostic or therapeutic purposes, ${ }^{2}$ so that almost all hospitalized patients at least once during their hospital stay and approximately one-quarter of them have been exposed to this procedure more than three times. ${ }^{3}$ This can cause pain and suffering for the patient, which can be a challenge for care and is still a common problem associated with blood sampling in clinical settings. ${ }^{4}$ Studies have shown high levels of acute pain, anxiety, stress, and irritability in patients of different ages following repeated vein punctures in one day to collect blood samples. ${ }^{5,6}$ In addition, the chance of vascular injury, bleeding, and nosocomial infection increases and makes future vascular use difficult ${ }^{7,8}$ and in rare cases causes peripheral nerve damage. ${ }^{9}$ It puts nurses at risk of unintended needle entry (needle stick). ${ }^{10}$ In some patients, such as those with chronic diseases, patients who have had multiple intravenous (IV) injections, IV drug abusers, patients with skin problems at the venous lines, patients taking anticoagulants, the elderly patients with fragile veins, and children, finding a suitable vein may require multiple vein punctures. ${ }^{11,12} \mathrm{IV}$ catheters are commonly used in most hospitalized patients for medical purposes such as injections of fluids, medications, and blood. ${ }^{11,14}$ These catheters make it unnecessary to use a needle and make it easier for patients with difficult venous blood sampling. ${ }^{13}$ This protects patients from repeated injuries by venous puncture sampling and nurses from needle puncture, providing comfort and time saving for patients and nurses, especially those in intensive and acute care, and suffer from collapsed veins, speeding up the results of tests and increasing the speed of medical and nursing care, reducing the risk of infections and other complications of blood sampling. ${ }^{11}$ Sampling for diagnostic tests using arterial and venous catheters has been controversial since half a century ago. ${ }^{15}$ The results of some studies have shown that by inserting and maintaining an IV catheter and accessing the patient's bloodstream, the blood sample needed for diagnostic tests can be obtained to reduce the complications of direct blood sampling., 5, ${ }^{10} \mathrm{On}$ the other hand, some studies have concluded that laboratory values obtained from IV catheter blood samples are invalid and recommend further studies in this area., ${ }^{5,13}$ On the other hand, the results of some studies suggest that the infusion intake through the IV catheter may affect the results of tests performed on IV catheter blood samples., ${ }^{7}$.

The present study aimed to compare the results of sodium, potassium, creatinine, and urea nitrogen levels in venous blood samples taken by direct blood sampling and peripheral venous catheter.

\section{Materials and Methods}

This analytical cross-sectional study was performed in 1396 (2017) from Poor-Sina Medical Center in Rasht. Sample size had $95 \%$ confidence interval and $90 \%$ test power according to 
the following sampling formula and based on similar studies 26 individuals were included in each group. ${ }^{16}$

$$
n=\frac{2\left(ख-\frac{\alpha}{2}+Z 1-\beta\right)^{2}(S D)^{2}}{d^{2}}
$$

Inclusion criteria included patients over 18 years of age with complete consciousness, with no coagulation problems, available peripheral veins, and peripheral venous with gauge20 catheter for up to 72 hours (receiving normal saline or dextrose saline or no infusion), who were prescribed by a physician who required blood sampling to check for blood sodium, potassium, creatinine, and urea nitrogen levels. Exclusion criteria included: patients with anemia and those with an IV arterial fistula and patients who had inoperable peripheral catheter or prolonged blood sampling more than 20 seconds for any reason and hemolyzed blood samples and actually.

Patients were divided into three groups ( 26 in each) based on the type of infusion that they received; Group 1: normal saline, Group 2: dextrose saline, and Group 3 did not receive any infusion and the catheter was heparin-locked.

Two separate blood samples were collected from each patient by one nurse, first sample from a peripheral vein catheter and the other from opposite hand direct vein blood sampling. ${ }^{16}$ In the first and second groups, serum therapy was stopped for 15 seconds. ${ }^{5}$ Then, in all three groups before blood sampling, the standard tourniquet was closed for 30 seconds, 5 $\mathrm{cm}$ above the peripheral venous catheter site. ${ }^{11,15}$ The volume of the catheter's dead space according to the size was set to $0.1 \mathrm{cc}$, and to ensure that, approximately $0.5 \mathrm{cc}$ of blood $(2.5$ times and more) was removed and discarded ${ }^{13}$ and by another syringe, $3 \mathrm{ml}$ was obtained for the test by the same route. Immediately after catheter blood sampling in all three groups, another $3 \mathrm{cc}$ blood samples were obtained from the opposite arm via direct blood sampling. After the sampling was completed, the IV fluid flow resumed as before. The test tubes were designated a code by researcher. The laboratory staff and statistical analyst were unaware of the coding and study groups. Samples were sent to one laboratory simultaneously to avoid possible variation between laboratories. Measurement of blood urea nitrogen (BUN) performed by using a diagnostic urea Bionic kit (Photometric - urea and alpha ketoglutarate dehydrogenase method - at a wavelength of $340 \mathrm{~nm}$ ) and creatinine was measured by Jaffe's method. Creatinine forms an amber yellow complex with alkaline picrate which was measured photometrically (Pars Azmoon Co., Tehran, Iran). Sodium and potassium were determined by ion selective electrodes. A database sheet was prepared which included the demographic information including age, gender, underlying diseases including diabetes, hypertension, and dyslipidemia, catheter fluid infusion rate, and type of received infusion, along with the results of laboratory tests.

\section{Data Analysis}

The data were analyzed using SPSS v18 software. Quantitative variables were expressed as mean \pm standard deviation and qualitative variables were expressed as frequency and percentage. Paired $t$-test was used to evaluate the differences between the two blood sampling methods and ANOVA or chi-square test was used to assess the differences between the three groups. $\mathrm{P}<0.05$ was considered significant.

\section{Ethical Considerations}

The present study was conducted under the auspices of the Research Ethics Committee of Guilan University of Medical Sciences (Approved Code: IR.GUMS.REC.1395 / 240) and the researchers adhered to all the principles of protocols and guidelines recommended by the Helsinki Convention on Ethics in Research. Prior to entering the study, informed consent was obtained from all participants. Study participants were assured that all patient information would be kept confidential and the outcome of the design would be generally published without mentioning the patient's name and any specifications. Patient participation in this study was completely voluntarily and no changes were made in the treatment or care of their patients due to their participation or not.

\section{Results}

In general, the youngest patient was 21 years old and the oldest was 90, with a mean age of 51.28 years. Analysis of the results among the three groups showed that there was no statistically significant difference in age between the three groups $(\mathrm{P}=0.689)$. Frequency of male gender was $61.5 \%(\mathrm{n}=16)$ in normal saline group, $65.4 \%(\mathrm{n}=17)$ in dextrose saline group, and 17 cases $(65.4 \%)$ in non-serum receiving group $(\mathrm{P}=0.946)$. Based on the history of underlying disease, it was found that overall 10 patients $(12.8 \%)$ with diabetes, 20 patients $(26.6 \%)$ with hypertension, and 10 patients $(12.8 \%)$ with dyslipidemia were included in this study. Although the frequency of diabetics was higher in the normal saline group $(\mathrm{P}=0.014)$, there was no statistically significant difference between the groups about the hypertension and dyslipidemia $(\mathrm{P}=0.625)$. There was no statistically differences between groups with respect to peripheral infusion rate $(\mathrm{P}=0.22)$ (Table 1$)$.

\section{Discussion}

The aim of the present study was to compare the results of sodium, potassium, creatinine, and urea nitrogen levels in venous blood samples obtained by direct blood sampling and peripheral venous catheter pathway, which were performed in order to achieve the primary and basic goals and to improve the quality of nursing care. The main aim is the reduction of pain and complications of direct blood sampling which patients may encounter during hospitalization.

The results of the present study showed that there was no statistically significant difference between the two methods of measuring BUN and serum creatinine between the study groups. According to the study of Hambleton et al in $2014^{10}$ and Zlotowski et al in $2001^{17}$ aiming comparison of laboratory tests through two methods of direct blood sampling and peripheral catheter and changes in laboratory results following IV normal saline injection indicated that the blood sampling method had no relationship with BUN and serum creatinine levels. Also, the results of BUN and serum creatinine following the two methods of blood sampling in patients receiving dextrose saline in the study of Ortels et al in $2014^{3}$ were not significantly different. In addition, the results of Yazdankhah Fard et al's study in $2014^{11}$ showed that there is no significant 


\begin{tabular}{|c|c|c|c|c|c|}
\hline & NS Group & DS Group & NL Group & Total & $P$ value \\
\hline \multicolumn{6}{|l|}{ Age } \\
\hline Age & $0 / 689^{*}$ & $51 / 28 \pm 18 / 95$ & $48 / 65 \pm 17 / 93$ & $52 / 88 \pm 18 / 77$ & $52 / 31 \pm 20 / 52 \#$ \\
\hline \multicolumn{6}{|l|}{ Sex } \\
\hline Male & $(\% 64 / 1) 50$ & $(\% 65 / 4) 17$ & $(\% 65 / 4) 17$ & $(\% 61 / 5) 16$ & \multirow{2}{*}{ 0/946\# } \\
\hline Female & $(\% 35 / 9) 28$ & $(\% 34 / 6) 9$ & $(\% 34 / 6) 9$ & $(\% 38 / 5) 10$ & \\
\hline \multicolumn{6}{|l|}{ Diabetes Hx } \\
\hline Yes & $(\% 12 / 8) 10$ & $(\% 11 / 5) 3$ & $(\% 0) 0$ & $(\% 26 / 9) 7$ & \multirow{2}{*}{ 0/014\# } \\
\hline No & $(\% 87 / 2) 68$ & $(\% \times 8 / 5) 23$ & $(\% 100) 26$ & $(\% 73 / 1) 19$ & \\
\hline \multicolumn{6}{|l|}{ HTN Hx } \\
\hline Yes & $(\% 25 / 6) 20$ & $(\% 19 / 2) 5$ & $(\% 26 / 9) 7$ & $(\%, 30 / 8) 8$ & \multirow{2}{*}{ 0/625\# } \\
\hline No & $(\% 74 / 4) 58$ & $(\% 80 / 8) 21$ & $(\% 73 / 1) 19$ & $(\% 69 / 2) 18$ & \\
\hline \multicolumn{6}{|l|}{ DLP Hx } \\
\hline Yes & $(\% 12 / 8) 10$ & $(\% 15 / 4) 4$ & $(\% 11 / 5) 3$ & $(\% 11 / 5) 3$ & \multirow{2}{*}{ 0/892\# } \\
\hline No & $(\% 87 / 2) 68$ & $(\% 84 / 6) 22$ & $(\% 88 / 5) 23$ & $(\% 88 / 5) 23$ & \\
\hline \multicolumn{5}{|c|}{ Venous Infusion Rate } & \multirow{5}{*}{ 0/217\# } \\
\hline $10 \mathrm{gtt} / \mathrm{min}$ & $(\% 44 / 2) 23$ & - & $(\% 53 / 9) 14$ & $(\%, 34 / 6) 9$ & \\
\hline $15 \mathrm{gtt} / \mathrm{min}$ & $(\% 30 / 8) 16$ & - & $(\% 19 / 2) 5$ & $(\% 42 / 3) 11$ & \\
\hline $20 \mathrm{gtt} / \mathrm{min}$ & $(\% 7 / 7) 4$ & - & $(\% 11 / 5) 3$ & $(\% 3 / 9) 1$ & \\
\hline $25 \mathrm{gtt} / \mathrm{min}$ & $(\% 17 / 3) 9)$ & - & $(\% 15 / 4) 4$ & $(\% 19 / 2) 5$ & \\
\hline
\end{tabular}

* ANOVA Test.

\# Chi-Square Test.

NS: Normal Saline Group, DS: Dextrose Saline Group, NL: No Infusion Group.

\begin{tabular}{|c|c|c|c|c|c|}
\hline Variables & Groups & $\begin{array}{c}\text { Direct } \\
\text { Sampling }\end{array}$ & $\begin{array}{l}\text { Catheter } \\
\text { Sampling }\end{array}$ & $\begin{array}{l}\text { Sampling Methods } \\
\text { Difference }\end{array}$ & $\begin{array}{l}\text { Significance } \\
\text { Level* }\end{array}$ \\
\hline \multirow{3}{*}{ BUN (mg/dl) } & NS & $17 / 31 \pm 7 / 81$ & $16 / 81 \pm 11 / 12$ & $-0 / 50 \pm 5 / 00$ & $0 / 614$ \\
\hline & DS & $15 / 15 \pm 7 / 72$ & $14 / 58 \pm 7 / 20$ & $-0 / 58 \pm 1 / 50$ & 0/061 \\
\hline & $\mathrm{NI}$ & $14 / 35 \pm 4 / 38$ & $14 / 46 \pm 3 / 93$ & $0 / 12 \pm 1 / 93$ & $0 / 762$ \\
\hline \multirow{3}{*}{$\mathrm{Cr}(\mathrm{mg} / \mathrm{dl})$} & NS & $1 / 10 \pm 0 / 59$ & $1 / 07 \pm 0 / 61$ & $-0 / 03 \pm 0 / 14$ & $0 / 337$ \\
\hline & DS & $1 / 05 \pm 0 / 36$ & $1 / 02 \pm 0 / 28$ & $-0 / 03 \pm 0 / 12$ & $0 / 153$ \\
\hline & $\mathrm{NI}$ & $1 / 02 \pm 0 / 17$ & $1 / 02 \pm 0 / 22$ & $0 / 00 \pm 0 / 11$ & 0/999 \\
\hline \multirow{3}{*}{$\mathrm{Na}(\mathrm{mEq} / \mathrm{L})$} & NS & $136 / 19 \pm 2 / 06$ & $137 / 81 \pm 2 / 42$ & $1 / 62 \pm 2 / 47$ & $0 / 003^{*}$ \\
\hline & DS & $135 / 54 \pm 2 / 56$ & $136 / 50 \pm 3 / 19$ & $0 / 96 \pm 2 / 86$ & 0/099 \\
\hline & $\mathrm{NI}$ & $136 / 96 \pm 2 / 46$ & $137 / 38 \pm 2 / 82$ & $0 / 42 \pm 2 / 02$ & 0/297 \\
\hline \multirow{3}{*}{$K(\mathrm{mEq} / \mathrm{L})$} & NS & $3 / 89 \pm 0 / 33$ & $3 / 96 \pm 0 / 44$ & $0 / 07 \pm 0 / 40$ & $0 / 386$ \\
\hline & DS & $3 / 73 \pm 0 / 52$ & $3 / 82 \pm 0 / 42$ & $0 / 08 \pm 0 / 39$ & $0 / 279$ \\
\hline & $\mathrm{NI}$ & $4 / 00 \pm 0 / 37$ & $4 / 29 \pm 0 / 63$ & $0 / 29 \pm 0 / 59$ & $0 / 020^{*}$ \\
\hline
\end{tabular}

* Paired-T Test, Significant level $P<0.05$

NS: Normal Saline Group, DS: Dextrose Saline Group, NI: No Infusion Group. 
difference in the results of these two parameters with blood sampling methods in patients receiving normal saline and dextrose saline, which is similar to the results of the present study. According to the results of the present study and the above-mentioned studies, it seems that the two methods of direct blood sampling and peripheral venous catheter path do not cause any differences in BUN and serum creatinine. The results of present study showed that sodium levels in the blood samples obtained from peripheral venous catheter was significantly higher than direct blood sampling in the group receiving normal saline. Blood potassium levels were higher after taking blood from peripheral venous catheter. In a study aimed comparing the results of biochemical and hematological tests out of two methods, routine blood sampling and peripheral venous catheter conducted by Gholipour et al. in $2016,{ }^{7}$ it was reported that there was a statistically significant difference between the results of patients' sodium samples. This finding is consistent with the results of the present study. Although another study in 2016, Ali Asgharpour et al., ${ }^{4}$ and Rezaei et al. ${ }^{18}$ showed that the difference in sodium and potassium levels between the two groups is not statistically significant. This is different from the results of our study. In these mentioned studies, the differences in sodium levels was not examined separately for types of infusion, while in the present study the type of infusion considered and higher sodium levels were achieved only in normal saline group. Also, in the study of Hamilton et al. in $2014,{ }^{10}$ Hamilton et al. reported that there is no significant difference in terms of these two electrolytes in patients receiving normal saline. In the present study, although results are similar to the mentioned study, there was no significant difference in potassium levels in patients receiving normal saline, but in general, sodium and potassium levels were significantly higher in the peripheral catheter blood sampling method. However, in the Hambleton study, normal saline injection was stopped for 2 minutes and $2 \mathrm{cc}$ of blood was discarded, while in the present study, the time of discontinuation of infusion, sampling and discarded blood volume was less, which could be the reason for differences in the results of these two studies. ${ }^{19}$ The effect of injectable drugs on test results was uncontrollable due to receiving through a peripheral catheter, which was one of the limitations of the present study. In addition, the time interval between two samples taken by the two methods may also affect the test results. So, a minimum time interval of about 1 minute was considered in this study. On the other hand, the present study has examined only four laboratory variables and its results cannot be generalized to all biochemical tests, so more studies in this field are needed for other laboratory tests. However, evaluation of the results of two blood sampling methods separately from the infusion received by patients is the strength of this study compared to similar studies in this field. The findings of this study can provide a basis for further studies on the effect of serum therapy on other tests of blood samples of the peripheral venous catheter in hospitalized patients aiming reduction of the complications of direct blood sampling.

\section{Conclusion}

The results of the present study showed that there was no statistically significant difference between the two methods of measuring BUN and creatinine between the study groups. However, blood sodium levels from peripheral venous catheter were significantly higher than direct blood sampling specifically in the normal saline group and also all cases. Blood potassium was higher in patients receiving saline dextrose as well as in total patients following peripheral venous catheter sampling. According to the results of this study and other studies, peripheral venous catheter blood sampling can be used to measure BUN and creatinine not for sodium and potassium regarding to the types of infusion.

\section{Acknowledgments}

The authors thank all people who helped us make this study and all colleagues in Guilan University of Medical Sciences for their invaluable suggestions during experimental design.

\section{Conflict of Interests:}

The authors of this manuscript have no conflicts of interest to declare.

\section{References}

1.Bernhofer El, St Marie B, Bena JF. A new clinical pain knowledge test for nurses: Development and psychometric evaluation. Pain Manag Nurs. 2017 Aug; 8(4):224-233

2.Yilmaz Kurt F, Aytekin Ozdemir A, Atay S. The effects of two methods on venipuncture pain in children: Procedural restraint and cognitive-behavioral intervention package. Pain Manag Nurs. 2019 Oct 15. pii: S15249042(18)30213-3.

3.Cadacio C, Nachamkin I. A novel needle-free blood draw device for sample collection from short peripheral catheters. J Infus Nurs. 2017:40(3):156-162.

4.Aliasgharpour M, Jafari R, Jalalinia S F, Jalal Madani S, Tabari F, Kazemnegad Lili A. Comparing blood values sampled from venipuncture and continuous infusion catheter. Crit Care Nurs J. 2016;9(3):1-5.

5.Ortells-Abuye N, Busquets-Puigdevall T, Díaz-Bergara M, Paguina-Marcos M, Sánchez-Pérez I. A cross-sectional study to compare two blood collection methods: direct venous puncture and peripheral venous catheter. BMJ Open. 2014;4(2):e004250

6.Kennedy RM, Luhmann J, Zempsky WT. Clinical implications of unmanaged needle-insertion pain and distress in children. Pediatrics. 2008 Nov;122(Suppl 3):S130-3.

7.Gholipour Baradari A, Zargar N, Aarabi M, Koohsari E, Emami Zeydi A. Comparison of hematologic and biochemical test results in blood samples obtained by venipuncture and peripheral intravenous catheter. J Mazandaran Univ Med Sci. 2016;26(139):66-72 (Persian).

8.Amini M, Vaseie M, Ansari I. The evaluation of nosocomial urinary tract infections and antimicrobial resistance in ICU patients, Tehran, Iran, 20122016. Acta Med Mediter, 2017;33:945-52

9.Asheghan M, Khatibi A, Holisaz MT. Paresthesia and forearm pain after phlebotomy due to medial antebrachial cutaneous nerve injury. J Brachial Plex Peripher Nerve Inj. 2011;6:5.

10. Hambleton VL, Gómez IA, Andreu FA. Venipuncture versus peripheral catheter: do infusions alter laboratory results? J Emerg Nurs. 2014 Jan; 40(1):20-6.

11. Yazdankhahfard M, Taghizadeganzadeh M, Farzaneh M, Mirzaei K. Comparison of biochemical laboratory values obtained by means of routine method of venipuncture versus peripheral intravenous infusion line after administration of fluids. Armaghane danesh. 2015;19(12):1021-1028 (Persian)

12. Braniff H, DeCarlo A, Haskamp AC, Broome ME. Pediatric blood sample collection from a pre-existing peripheral intravenous (PIV) catheter. J Pediatr Nurs. 2014 Sep-Oct;29(5):451-6.

13. Mofrad ZP, Shafiee S, Mahmoodi N, Jahantigh M, Samadzadeh H. Comparison of the effect of direct venipuncture and venous catheter blood sampling methods on the biochemical results of patients hospitalized in CCU. Med Surg Nurs J. 2016;5(1):11-5. 
14. Cicolini G, Manzoli L, Simonetti V, Flacco ME, Comparcini D, Capasso L, et al. Phlebitis risk varies by peripheral venous catheter site and increases after 96 hours: a large multicentre prospective study. J Adv Nurs. 2014 Nov;70(11):2539-49.

15. Aghaei-Hashjin M, Javadi-Pashaki N, Kazemnezhad-Leyli E, AghajaniNasab M. Comparison of the results of blood sugar between direct blood sampling and peripheral catheter in hospitalized patients. J Biochem Tech. 2018;Special Issue (2):189-195.

16. Watson KR, O'kell RT, Joyce JT. Data regarding blood drawing sites in patients receiving intravenous fluids. Am J Clin Pathol. 1983 Jan;79(1):119-21.
17. Zlotowski SJ, Kupas DF, Wood GC. Comparison of laboratory values obtained by means of routine venipuncture versus peripheral intravenous catheter after a normal saline solution bolus. Ann Emerg Med. 2001 Nov;38(5):497504.

18. Rezaei K, Kohestani H, Zand S. Comparison of biochemistry values obtained by venipuncture and saline lock after intermittent administration of fluids and drugs. J Arak Uni Med Sci. 2009;12(1):49-56 (Persian).

19. Baker RB, Summer SS, Lawrence M, Shova A, McGraw CA, Khoury J.

Determining optimal waste volume from an intravenous catheter. J Infus Nurs. 2013;36(2):92-96.

This work is licensed under a Creative Commons Attribution-NonCommercial 3.0 Unported License which allows users to read, copy, distribute and make derivative works for non-commercial purposes from the material, as long as the author of the original work is cited properly. 